
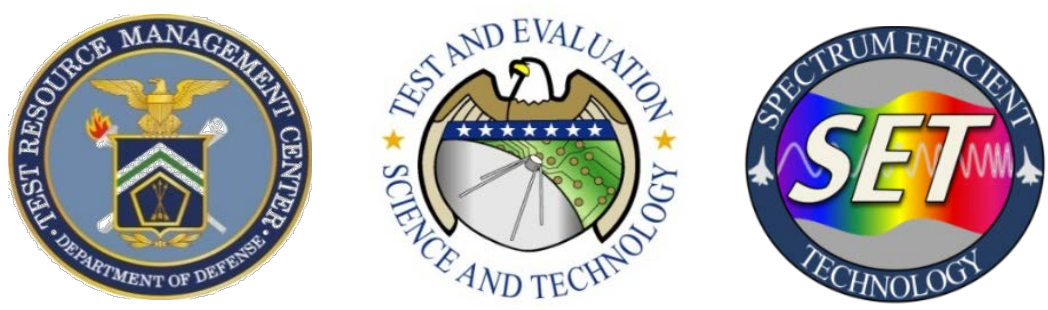

\title{
Evaluating CMA Equalization of SOQPSK-TG for Aeronautical Telemetry
}

\section{March 2015}

Tom Young

SET Executing Agent

412 TENG/ENI

(661) 277-1071

Email: tommy.young.1@us.af.mil

DISTRIBUTION STATEMENT A. Approved for public release: distribution unlimited.

Test Resource Management Center (TRMC)

Test \& Evaluation/ Science \& Technology (T\&E/S\&T)

Spectrum Efficient Technology (SET) 


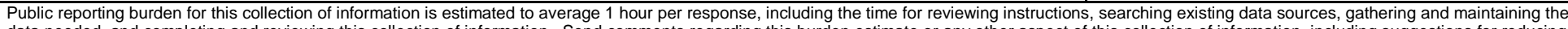

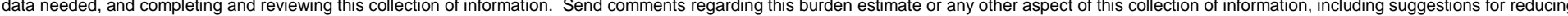

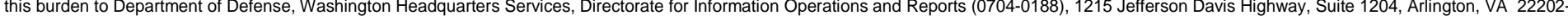

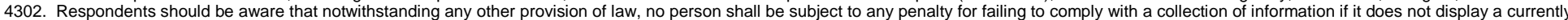
valid OMB control number. PLEASE DO NOT RETURN YOUR FORM TO THE ABOVE ADDRESS.

\begin{tabular}{l|l} 
1. REPORT DATE $(D D-M M-Y Y Y Y)$ & 2. REPORT TYPE
\end{tabular}

\begin{tabular}{l|l} 
21-04-2015 & Technical Paper \\
\hline
\end{tabular}

4. TITLE AND SUBTITLE

Evaluating CMA Equalization of SOQPK-TG Data for Aeronautical Telemetry

3. DATES COVERED (From - To)

5a. CONTRACT NUMBER:

W900KK-13-C-0026

5b. GRANT NUMBER: N/A

6. AUTHOR(S)

5c. PROGRAM ELEMENT NUMBER

Arlene Cole-Rhodes, Serge KoneDossonguia, Henry Umuoloa (MSU)

Michael Rice (BYU)

5d. PROJECT NUMBER

7. PERFORMING ORGANIZATION NAME(S) AND ADDRESS(ES)
Electrical \& Computer Engineering Dept., Morgan State University, Baltimore, MD, USA

21251

5e. TASK NUMBER

5f. WORK UNIT NUMBER

8. PERFORMING ORGANIZATION REPORT NUMBER

412TW-PA-15187

Brigham Young University, A-41 ASB, Provo, UT 84602-1014

9. SPONSORING I MONITORING AGENCY NAME(S) AND ADDRESS(ES)

Test Resource Management Center

Test and Evaluation/ Science and Technology

4800 Mark Center Drive, Suite 07J22, Alexandria, VA 22350

10. SPONSOR/MONITOR'S ACRONYM(S)

$\mathrm{N} / \mathrm{A}$

11. SPONSOR/MONITOR'S REPORT

NUMBER(S) SET 2015-0036

12. DISTRIBUTION I AVAILABILITY STATEMENT

Approved for public release A: distribution is unlimited.

\section{SUPPLEMENTARY NOTES}

CA: Air Force Flight Test Center Edwards AFB CA CC: 012100

\section{ABSTRACT}

This paper presents the results of using a constant modulus algorithm (CMA) to recover shaped offset quadrature-phase shift keying (SOQPSK)-TG modulated data, which has been transmitted using the iNET data packet structure. This standard is defined and used for aeronautical telemetry. Based on the iNET-packet structure, the adaptive block processing CMA equalizer can be initialized using the minimum mean square error (MMSE) equalizer [3]. This CMA equalizer is being evaluated for use on iNET structured data, with initial tests being conducted on measured data which has been received in a controlled laboratory environment. Thus the CMA equalizer is applied at the receiver to data packets which have been experimentally generated in order to determine the feasibility of our equalization approach, and its performance is compared to that of the MMSE equalizer. Performance evaluation is based on computed bit error rate (BER) counts for these equalizers.

\section{SUBJECT TERMS}

MMSE equalizer, adaptive CMA equalizers, SOQPSK modulation, iNET packet structure, aeronautical telemetry channel, Spectrum.

16. SECURITY CLASSIFICATION OF:

Unclassified

\section{a. REPORT}

Unclassified

\section{b. ABSTRACT}

Unclassified

\section{c. THIS PAGE}

Unclassified
17. LIMITATION OF ABSTRACT

None
18. NUMBER OF PAGES 19a. NAME OF RESPONSIBLE PERSON 412 TENG/EN (Tech Pubs)

19b. TELEPHONE NUMBER (include area code) 


\title{
Evaluating CMA equalization of SOQPK-TG Data for aeronautical telemetry
}

\author{
Arlene Cole-Rhodes*a ${ }^{\mathrm{a}}$, Serge KoneDossongui ${ }^{\mathrm{a}}$, Henry Umuolo ${ }^{\mathrm{a}}$, Michael Rice $^{\mathrm{b}}$ \\ ${ }^{a}$ Electrical \& Computer Engineering Dept., Morgan State University, Baltimore, MD, USA 21251; \\ ${ }^{\mathrm{b}}$ Brigham Young University, Provo, UT, USA 84602.
}

\begin{abstract}
This paper presents the results of using a constant modulus algorithm (CMA) to recover shaped offset quadrature-phase shift keying (SOQPSK)-TG modulated data, which has been transmitted using the iNET data packet structure. This standard is defined and used for aeronautical telemetry. Based on the iNET-packet structure, the adaptive block processing CMA equalizer can be initialized using the minimum mean square error (MMSE) equalizer [3]. This CMA equalizer is being evaluated for use on iNET structured data, with initial tests being conducted on measured data which has been received in a controlled laboratory environment. Thus the CMA equalizer is applied at the receiver to data packets which have been experimentally generated in order to determine the feasibility of our equalization approach, and its performance is compared to that of the MMSE equalizer. Performance evaluation is based on computed bit error rate (BER) counts for these equalizers.
\end{abstract}

Keywords: MMSE equalizer, adaptive CMA equalizers, SOQPSK modulation, iNET packet structure, aeronautical telemetry channel.

\section{INTRODUCTION}

We investigate the effectiveness of a CMA equalizer [1] in recovering an unknown data bit-stream that has been transmitted over an unknown channel using the iNET data packet structure, with shaped offset QPSK, version 'TG' (SOQPSK-TG)modulation. Previous work by Law [2] had noted some difficulties with the use of CMA to equalize SOQPSK-TG over a telemetry channel, but subsequent work using a simulated aeronautical channel $([3,4,5])$ has shown that a block processing CMA equalizer can be an effective equalizer for recovering SOQPSK-TG modulated signals transmitted with the iNET data packet structure. This equalizer should be especially effective in the case that the known data bits contained in the iNET-packet structure (i.e. the preamble and asynchronous marker (ASM) bits) are leveraged to provide an alternative method of initializing the CMA equalizer. It has been shown in [3] that compared to the usual method of using center-tap initialization, the convergence time of the adaptation can be decreased and a lower final bit error count is produced when CMA is initialized using the minimum mean square error (MMSE) equalizer. For a realtime application, the block processing CMA equalizer must be adapted while receiving the data packet stream and the bit error rate will be used as the basis for evaluation and comparison. For the simulated experiments the bit error count is noted after convergence of the equalizer is achieved, and the update of the CMA weight vector continues using each received data packet while accumulating errors. For the measured data experiments the bit error count is noted for every packet starting from the first packet.

\section{COMMUNICATION DATA STRUCTURE}

The INET packet structure is made up of a known preamble of length 128 bits, and ASM is of length 64 bits, together with the actual data of length 6144 bits. The total packet length is 6336 bits and this packet structure is shown in Fig. 1.

*arlene.colerhodes@morgan.edu; phone 1443 885-4207; morgan.edu 


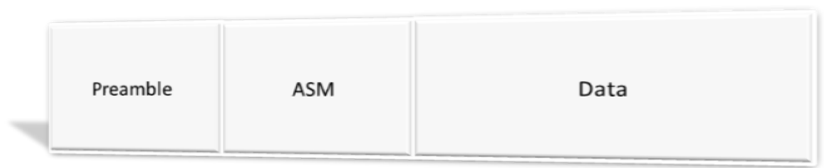

Fig. 1: INET Packet structure

The communication system accepts this structured bit stream, which is formatted using the iNET packet structure of Fig. 1 , at the transmitter. It is then modulated using SOQPSK-TG which produces a signal $s(t)$ of the form:

$$
s(t)=\sqrt{2 E / T} \cos \left[2 \pi f_{o} t+\phi(t, \alpha)+\phi_{o}\right]
$$

with phase given by

$$
\phi(t, \alpha)=2 \pi h \int_{-\infty}^{t} \sum_{i=-\infty}^{+\infty} \alpha_{i} g(\tau-i T) d \tau
$$

The SOQPSK pulse, $g(t)$ is a windowed impulse response of the spectral raised cosine which is proportional to frequency; $h=0.5$ is the modulation index; $E$ is the bit energy; and $T$ is the bit sampling interval. This modulation scheme involves the use of a precoder which converts the binary data into a ternary output stream $\alpha_{i}$ consisting of $\{-1,0$, $+1\}$ together with the use of differential encoding. Thus the transmitted signal SOQPSK-TG is a constrained partialresponse continuous phase modulation (CPM) with a ternary alphabet and its parameters are described in detail in $[6,7]$.

The samples of the SOQPSK-TG modulated carrier signal are transmitted over an unknown AWGN channel, determined by the specific laboratory environment. The maximum length of the channel is assumed to be 37 , and the SOQPSK-TG signal experiences multipath propagation over this unknown channel. For our experiments the transmitted data is perfectly known, and so we are able to determine and count the number of bit errors after the equalizer has been applied to the received data packets. We note that received data packets are blocks of length $N$, which are at least 12672 samples, since the modulator operates at an equivalent rate of two samples per bit. The equalizer for the received signal is an FIR filter of impulse response, $w(n)$. The equalizer output is processed by a symbol-by-symbol SOQPSK-TG demodulator [6] to produce the bit estimates, which determine the error count.

Using the known preamble and ASM databits contained within the transmitted data packet, it is possible to compute the minimum mean square error (MMSE) and zero-forcing equalizers for each received data packet. Thus for efficiency it has been proposed in [3] that one of these be used to initialize the CMA equalizer in place of the usual center tap initializer. The CMA equalizer is then be updated iteratively once per received data packet, and bit error rates are computed.

\section{CMA EQUALIZATION ALGORITHM}

The constant modulus algorithm (CMA) is a blind equalizer, which is adaptive and minimizes a specific cost function. The CMA cost function is given by

$$
J_{C M A}(y(n))=E\left\{\left(|y(n)|^{2}-R_{2}\right)^{2}\right\}
$$

where the radius $R_{2}$ is determined based on the known modulation type used for signal transmission. For SOQPSK, the transmitted signal is CPM and of the form: $s(n)=\exp (j \phi(n))$ so the value of CMA radius used is $R_{2}=1$. Thus SOQPSK-TG is constant modulus, and by simulation CMA equalization has been shown to work well on this modulation type [3,4]. Based on the iNET packet structure the MMSE equalizer can be applied to recover the received 
data, and by initializing CMA with the MMSE equalizer there will be no phase ambiguity in the CMA equalized symbols for the data packet to which it is applied.

The iterative update of the equalizer weights for the CMA cost function is based on steepest descent, and is given by

$$
\underline{w}_{p+1}=\underline{w}_{p}-\mu \nabla_{w} J_{C M A}(y(n))
$$

where $\underline{w}_{p}$ is the equalizer weight vector and $\mu=5 \mathrm{e}-2$ is a fixed step-size. The gradient vector of the cost function is given as:

$$
\nabla_{w} J_{C M A}(y(n))=E\left\{4\left(|y(n)|^{2}-R_{2}\right) y(n) \underline{x}_{n}{ }^{*}\right\}
$$

where the $*$ operator denotes the complex conjugate. For our implementation of CMA the starting equalizer weight vector, $\underline{w}_{0}$ is computed based on the minimum mean square (MMSE) equalizer. We will investigate the performance of this CMA equalizer when it is applied at the receiver to recover the transmitted data packets.

\section{EXPERIMENTAL SYSTEM IMPLEMENTATION}

In this work, the performance of the CMA equalizer will also be evaluated using a data stream produced by the experimental setup shown in Fig. 2. The SOQPSK-TG transmitter was set to $10 \mathrm{Mbits} / \mathrm{s}$ using an internally generated length-2047 PN sequence. (With the addition of the preamble and ASM fields, the over-the-air rate was 10.3125 Mbits/s.) The signal was attenuated and transmitted over the air in a laboratory whose walls and desks created ample opportunities for short-delay multipath, typical of that encountered on aeronautical test ranges [8]. The received signal was filtered and down-converted to a $70 \mathrm{MHz}$ IF where it was sampled at $100 \mathrm{Msamples/s}$. The sample sequence was stored on a hard drive where MATLAB ${ }^{\circledR}$ was used to create the low-pass equivalent signal at a sample rate equivalent to 2 samples/bit.

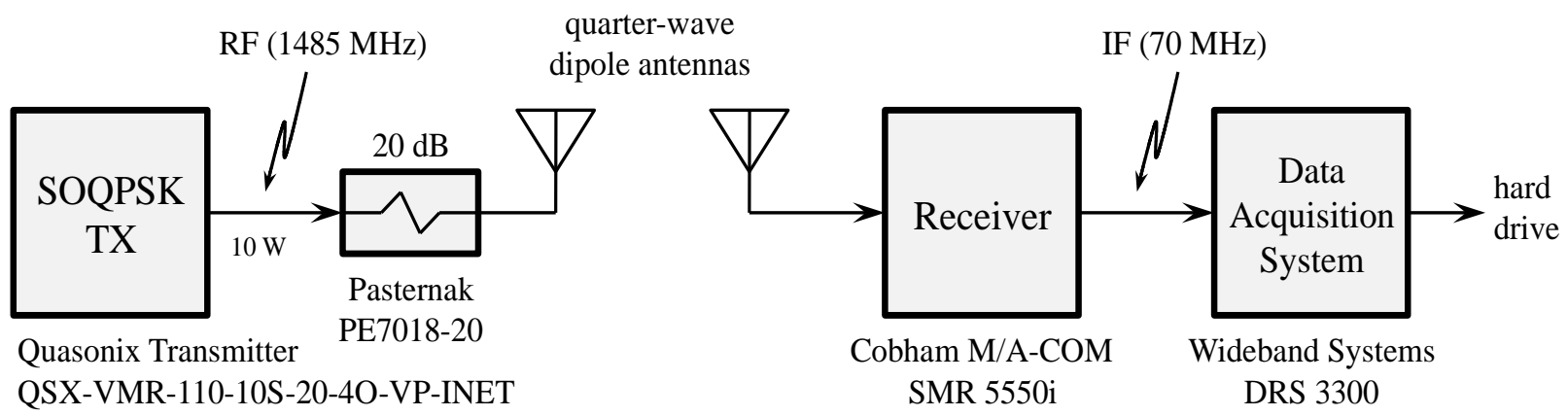

Fig. 2: Experimental setup used to generate measured data

For our experiments, both the MMSE and CMA equalizers are applied and evaluated at the receiver. Any frequency offset which may have been introduced by the channel is estimated here and is first removed from the received samples before the equalizer is computed and applied. The preamble data in the first packet is used to estimate the channel, and the SNR for this data set is also estimated. The SNR for the received data set was estimated to be $25 \mathrm{~dB}$. We note that for CMA, the MMSE equalizer is calculated for the first data packet only and used only as an initializer. After initialization, the equalizer filter coefficients are updated once for each received packet using CMA. For comparison purposes we can calculate the MMSE equalizer for each received data packet. A first-order phase lock loop (PLL) is used and it operates in a decision-directed mode. Outlined below are details of the data processing steps that are used at the receiver. 


\section{$\underline{\text { Receiver Data Processing and Equalization Procedure }}$}

Step 1: Load received iNET data packets.

Step 2: Find the preamble bits.

Step 3: Estimate the frequency offset, and derotate the received samples.

Step 4: Initialize the CMA equalizer filter using MMSE, and apply to the data.

Step 5: Update the CMA equalizer filter coefficients once (for current data packet).

Step 6: Apply a detection filter and downsample data.

Step 7: Apply phase lock loop (PLL) and record bit error for current packet

Step 8: Repeat Steps 5 through 7 for each received packet.

Step 9: Compute the final overall bit error rate $(B E R)$ for data, by correlation with the transmitted PN sequence.

\section{EXPERIMENTAL RESULTS}

\subsection{Simulation Experiments}

Our proposed CMA equalization procedure was first evaluated by simulation. An iNET data packet containing random data as well as the preamble and ASM specified by the iNET packet structure, was generated at 2 samples per bit, and modulated by SOQPSK-TG. The performance of the proposed equalization scheme was evaluated using a simulated aeronautical test channel, which represents the flight taxiway before take-off. The spectrum of this channel is shown in Fig. 3 together with that of the SOQPSK modulated signal. This packet was sent through a simulated aeronautical test channel and noise was added to create the received data packet to be equalized. The signal transmission process for this iNET data packet at $25 \mathrm{~dB}$ SNR is shown in Fig. 4.

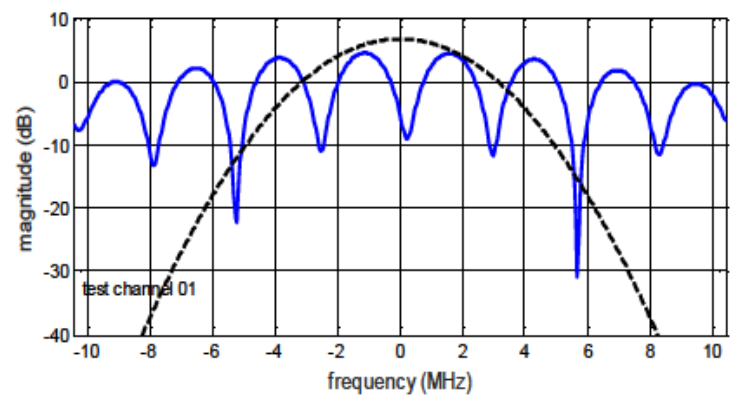

Fig. 3: Frequency Response of Channel 1 (taxiway)

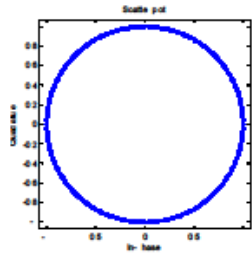

Channel
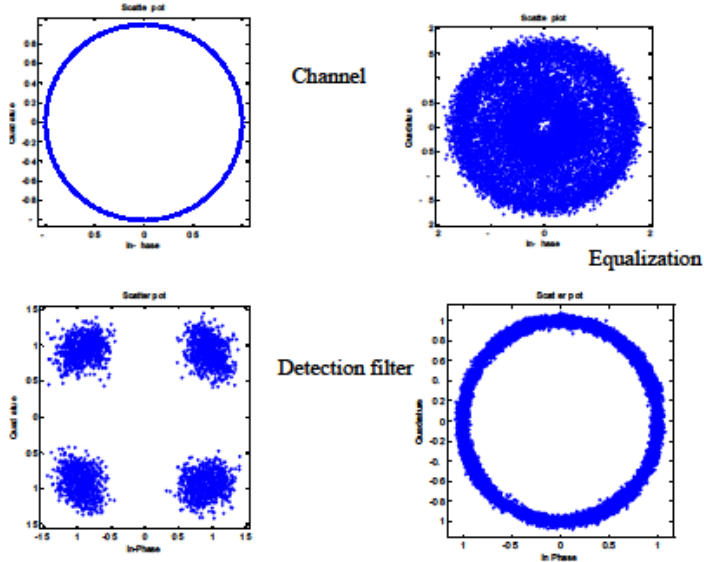

Fig. 4: SOQPSK Signal Transmission (simulated)

Focusing on Fig. 4, we note that the transmitted SOQPSK modulated signal lies on a circle of unit radius. The effect of multipath on the received data, is seen in the top right figure of Fig. 4, and the effect of CMA equalizer is shown below this on the bottom right. The bottom left of Fig. 4 shows the resulting QPSK symbols after the detection filter is applied to the equalized data bits. 
Fig. 5 shows the BER performance of the proposed MMSE-initialized CMA equalizer for two different scenarios. The baseline curve (triangles) is obtained by updating the CMA equalizer using a single iNET data packet, following initialization by the MMSE equalizer. This test was performed to establish the feasibility of the approach, and provide an indication of the 'best' possible performance to be expected. The second curve (circles) is obtained by simulating a more realistic real-time experiment in which the received data is processed block-by-block $(\mathrm{BxB})$ with a single update of the CMA equalizer coefficients for each received data packet. This is similar to the processing method which is to be used with the measured data. The theoretical curve for AWGN (with no multipath) is included, as well as that obtained from applying the MMSE equalizer to this data set.

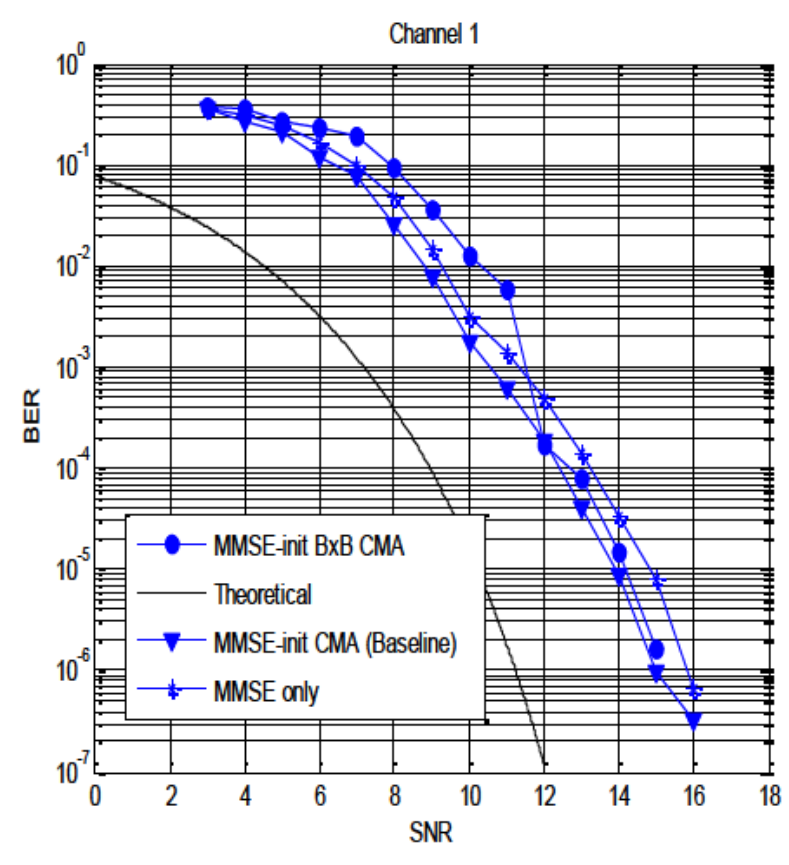

Fig. 5: Comparing Equalizer Performance

These simulation experiments show that the CMA performance is very close to that of the MMSE equalizer (with one $\mathrm{dB}$ performance improvement), and it indicates the feasibility of the proposed real-time CMA equalization approach for this SOQPSK data.

\subsection{Experiments with Measured Data}

Now to focus on equalization of the measured data obtained from the experimental setup of section 4 , since the transmitted data is a known $2047 \mathrm{PN}$-sequence, the number of errors can be determined and counted by correlation with this known sequence. The output of PN-correlator (step 9), which is used to count errors after equalization of our experimentally measured data, is shown in Figs. 6 (a) and (b) for the first nine data packets of the MMSE and CMA equalizers respectively. We observe from Fig. 6(a) that the MMSE equalizer produces zero errors, while in Fig. 6(b) corresponding to the CMA equalized data, we note the occurrence of some very clear inversions of the data symbols, which leads to very large error counts. 


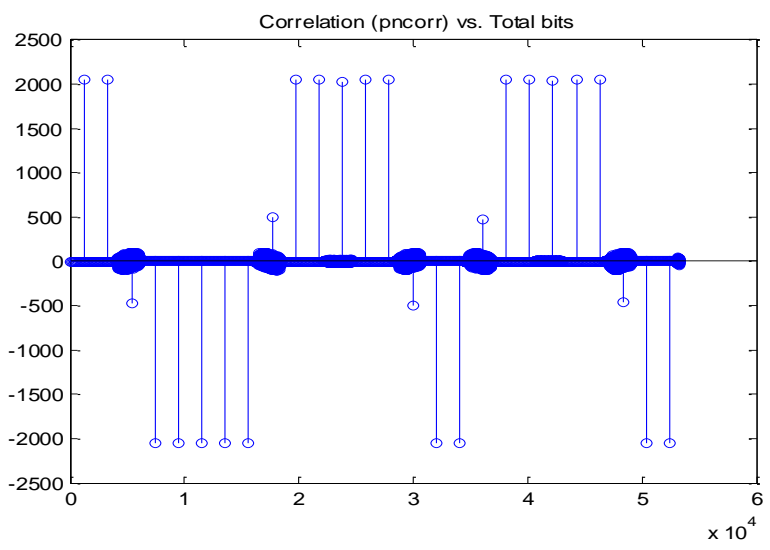

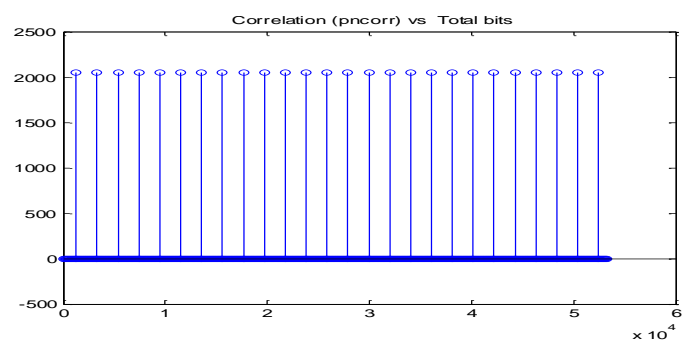

Fig. 6(a): Correlator output for MMSE Equalizer

Fig. 6(b): Correlator output for CMA Equalizer (9 packets)

Additional analysis of the results of Fig 6(b) shows that the symbol inversions (with resulting errors) occur always at the start of each new data packet, which is approximately every 3 spikes of 2047. We note that for SOPQSK, the detected symbols resemble QPSK which looks the same for any phase rotation which is a multiple of 90 degrees. By numbering the QPSK symbol locations as 1 through 4 to correspond to the symbol ordering $\{1+\mathrm{j} ;-1+\mathrm{j} ; 1-\mathrm{j} ;-1-\mathrm{j}\}$, the position of the last ASM symbol (number 32) in the iNET packet is known and should be at location 1. But our investigation has revealed that when the CMA equalizer is applied to a new data packet the detected symbols from the equalized data may be rotated by some multiple of pi/2 out of phase from the correct position. A sample of these results is shown in Table 1 , which provides the new location of the last (known) ASM symbol after CMA equalization, for each of packets 1 through10. We note that this symbol location changes nearly every time a new data packet is equalized by CMA, indicating that a phase rotation is also being introduced by the CMA equalizer. This finding should not be totally unexpected since CMA is known to be a phase blind equalizer.

Table 1. Location of last ASM symbol $=1$.

\begin{tabular}{|l|l|l|l|l|l|l|l|l|l|l|}
\hline Packet No. & $\mathbf{1}$ & $\mathbf{2}$ & $\mathbf{3}$ & $\mathbf{4}$ & $\mathbf{5}$ & $\mathbf{6}$ & $\mathbf{7}$ & $\mathbf{8}$ & $\mathbf{9}$ & $\mathbf{1 0}$ \\
\hline $\begin{array}{l}\text { New ASM } \\
\text { Location }\end{array}$ & 1 & 4 & 4 & 1 & 3 & 4 & 2 & 3 & 3 & 2 \\
\hline
\end{tabular}

As we continue to develop a solution to this problem, we will use the phase information provided by Table 1 to determine and apply a CMA phase correction for each data packet. It is our hope that the phase lock loop will be more effective in tracking once the additional phase error introduced by CMA has been corrected for. In addition we expect that an adaptive equalizer such as CMA will be more effective than a fixed equalizer (such as the MMSE equalizer) in tracking a time-varying aeronautical channel.

\section{CONCLUSIONS}

In this work we have applied an MMSE-initialized CMA equalizer to simulated SOQPSK data, and we have investigated its effectiveness for measured SOQPSK modulated data which has been transmitted in laboratory environment using the iNET packet structure. One conclusion from this initial investigation is that the phase blind property of CMA makes it non-ideal for a straightforward equalization of SOQPSK modulated data. But because of the known ASM and preamble data bits contained in the iNET packet structure, it is expected that we will be able to leverage some additional information about the received data, in order to properly equalize this data using CMA. 


\section{ACKNOWLEDGEMENTS}

This work was funded by the Test Resource Management Center (TRMC) Test and Evaluation Science and Technology (T\&E/S\&T) Program through the U.S. Army Program Executive Office for Simulation, Training and Instrumentation (PEO STRI) under contract W900KK-13-C-0026 (PAQ).

\section{REFERENCES}

[1] Johnson, R., et. al. "Blind equalization using the constant modulus criterion: A review", Proceedings of IEEE 86(10), 1927-1950 (1998).

[2] Law, E., "How well does a blind adaptive CMA equalizer work in a simulated telemetry multipath environment", Proc. International Telemetry Conference (2004).

[3] Cole-Rhodes, A., Umuolo, H., Moazzami, F., Rice, M.,"A block processing approach to CMA equalization of SOQPSK for aeronautical telemetry", Proc. IEEE Military Communications Conference, (2014).

[4] Rice, M., Saquib, M., Afran, M.S., Cole-Rhodes, A., Moazzami, F., "On performance of equalization techniques for aeronautical telemetry", Proc. IEEE Military Communications Conference, (2014).

[5] Rice, M., "Phase 1 Report: Preamble assisted equalization for aeronautical telemetry (PAQ)," Brigham Young University, Technical Report, 2014, submitted to the Spectrum Efficient Technologies (SET) Office of the Science \& Technology, Test \& Evaluation (S\&T/T\&E) Program, Test Resource Management Center (TRMC). Also available online at <http://hdl.lib.byu.edu/1877/3242> (2014).

[6] Nelson, T., Perrins, E., Rice, M., "Near optimal common detection techniques for shaped offset QPSK and Feher's QPSK," IEEE Transactions on Communications 56(5), 724-735 (2008).

[7] Perrins, E., "FEC systems for aeronautical telemetry," IEEE Transactions on Aerospace and Electronic Systems 49(4), 2340-2352, (2013).

[8] Rice, M., Davis, A., and Bettweiser, C., "A wideband channel model for aeronautical telemetry," IEEE Transactions on Aerospace and Electronic Systems 40(1), 67-69, (2004). 